\title{
EXPERIMENTAL AND NUMERICAL VIBRATION ANALYSIS OF PRINTED CIRCUIT BOARDS
}

\author{
Richard Bachoo $^{*}$, Shurland Balliram², Jacqueline Bridge ${ }^{3}$ \\ ${ }^{1,2,3}$ Faculty of Engineering, The University of the West Indies, Trinidad \\ ${ }^{1}$ Email: Richard.Bachoo@ sta.uwi.edu*(Corresponding author) \\ ${ }^{2}$ Email: Shurland.Balliram@my.uwi.edu \\ ${ }^{3}$ Email: Jacqueline.Bridge@ sta.uwi.edu
}

\begin{abstract}
Printed circuit boards (PCBs) are important modules which are incorporated in a wide range of industrial equipment and machinery for the purpose of control or signal manipulation applications. PCBs situated in dynamic environments may be prone to failure from excessive amounts of cyclical stresses arising from harmonic or random vibration sources. The ability to numerically model and predict the dynamic behaviour of PCBs and associated components is therefore a valuable tool for analysts concerned with PCB reliability. In this paper, experimental vibration analysis and the finite element method (FEM) are used to investigate the changes in resonant behaviour of a PCB as the mass, location and stiffness of electronic components vary. Circuit boards that are either sparsely or densely populated with ubiquitous soldered electronic components such as resistors, transistors, capacitors and integrated circuits are considered. The analysis indicates that for boards with a small number of components the natural frequency decreases compared to that of the bare PCB whilst a board with a larger number of soldered components has a corresponding increase. It is also shown that the overall effect of the solder is to reduce the natural frequency of the PCB and to a lesser extent the damping ratio. The study identifies the potential of tailoring the vibration response of a PCB by the appropriate selection and location of its connected components.
\end{abstract}

Keywords: Electrodynamic shaker, Experimental modal analysis, Finite element method, Harmonic vibration, Printed circuit board, $P C B$.

https://doi.org/10.47412/UMTW9840

\section{Introduction}

Electronic and electrical systems perform vital functions for a range of machinery and instruments in industries including the avionics, marine and automotive sectors. The printed circuit board (PCB) remains the primary infrastructure which supports and connects the electrical and electronic components needed to carry out such functions. In PCBs where the components are surface mounted, the two main factors which affect reliability of the package are thermal cycling and vibration induced fatigue. In the case of thermal cycling the soldered joints are most prone to failure, whereas when structural vibration is of concern, the component leads are most vulnerable [1]. This work is concerned with vibratory response as it is often the case that PCBs are installed in locations where vibration levels may be high enough to result in failure. The sources of excitation may be harmonic or random in nature and predicting the response levels of the board and its components is ultimately required to calculate whether induced stress levels are beyond some predetermined threshold. Induced stresses are often at their highest levels when the board is forced to vibrate at its resonant frequency. 
The approaches used to model the dynamic behaviour of PCBs include direct analytical methods [2,3], the finite element method [4,5] and experimental vibration analysis [6,7]. Owing to the complex nature of PCBs direct analytical methods have limited application and the finite element method is often the preferred choice for modelling. A wide range of literature have focused on using FEM for the fatigue life estimation of PCBs and their components. Ref. [8] merged the FEM and experimental harmonic vibrational analysis to determine S-N curves for Plastic Ball Grid Array (PBGA) solder balls. Ref. [9] used FEM in conjunction with rainflow counting to determine the fatigue life of thin core ball grid arrays (CTBGAs) and Zhou et al., [10] conducted harmonic and random vibration durability tests for SAC305 and Sn37Pb solder alloys. Ref. [11] examined the component leads in detail using the FEM and showed that the fundamental frequency of the soldered lead is at a minimum five times higher than that of the unsoldered lead. In certain applications a detailed FEM including all attached components is replaced by a smeared approach whereby the localized inhomogeneities in mass and stiffness are averaged over the entire board [12].

Fundamentally, the fatigue life associated with a PCB package is directly related to the modal characteristics of the board and its attached components. Ref. [13] performed experimental modal analysis on a PCB with and without a single surface mounted component and showed that the latter had slightly lower natural frequencies than the former. In this work we examine, experimentally and numerically, the variations in natural frequencies of a PCB as the board gradually becomes densely populated with surface mounted components. It is shown that the location and mass of the electronic components soldered on a board can strongly influence the resonant mode of vibration and the inherent damping. For boards with a small number of components the natural frequency decreases compared to that of the bare PCB which is in agreement with Ref. [13], however, a board with a larger number of soldered components has a corresponding increase in natural frequency owing to the increase in local stiffness. In each case detailed finite element models are generated to produce response levels which agree well with experimental data. This emphasizes the value of using numerical models at the design stage to adequately select and locate components on a PCB to acquire the desired response levels.

\section{Description of the PCB and Surface Mounted Components}

The selected PCB functions as an adjustable direct current (DC) regulated power supply when built-up with all the soldered components. The PCB is constructed from FR4 and its dimensions and properties are as shown in Table 1. The chosen solder material is lead-free SAC305 and the leads for the electronic components are all copper. Material properties of the solder and leads are also stated in Table 1. The mass and tag of each surface mounted component is given in Table 2 whilst the location on the bare PCB is shown in Fig. 1a.

Table 1. Properties of the bare printed circuit board (PCB) and solder.

\begin{tabular}{cccccc}
\hline Item & Material & $\begin{array}{c}\text { Dimensions } \\
\mathrm{mm} \times \mathrm{mm} \times \mathrm{mm}\end{array}$ & $\begin{array}{c}\text { Density } \\
\left(\mathrm{kg} / \mathrm{m}^{3}\right)\end{array}$ & $\begin{array}{c}\text { Young's } \\
\text { Modulus }(\mathrm{GPa})\end{array}$ & $\begin{array}{c}\text { Poisson } \\
\text { Ratio }\end{array}$ \\
PCB & FR4 & $84 \times 84 \times 1.5$ & 1970 & 17.2 & 0.40 \\
Solder & SAC305 & - & 8420 & 53.3 & 0.35 \\
Leads & Copper & - & 8960 & 128 & 0.34 \\
\hline
\end{tabular}

Table 2. Tags and masses of surface mounted components.

\begin{tabular}{|c|c|c|c|c|c|}
\hline Component & Tag & Mass(g) & Component & Tag & Mass (g) \\
\hline Resistors & & & Integrated c & & \\
\hline $82 \mathrm{Ohm}$ & 82 & 0.1 & TL081 & TL081 & 0.471 \\
\hline $22 \mathrm{Ohm}$ & 22 & 0.1 & Diodes & & \\
\hline
\end{tabular}


Faculty of Engineering, The UWI, St. Augustine | June $1^{\text {st }}-5^{\text {th }}, 2020$

\begin{tabular}{|c|c|c|c|c|c|}
\hline $10 \mathrm{~K} \mathrm{Ohm}$ & $10 \mathrm{~K}$ & 0.1 & $1 \mathrm{~N} 4004$ & 4004 & 0.3 \\
\hline $56 \mathrm{~K} \mathrm{Ohm}$ & $56 \mathrm{~K}$ & 0.1 & 1N5408 & 1N5408 & 0.725 \\
\hline 27K Ohm & $27 \mathrm{~K}$ & 0.1 & $1 \mathrm{~N} 4148$ & 4148 & 0.0912 \\
\hline $2.2 \mathrm{~K} \mathrm{Ohm}$ & $2 \mathrm{~K} 2$ & 0.1 & $5.1 \mathrm{~V} \mathrm{ZD}$ & $5 \mathrm{~V} 1$ & 0.0912 \\
\hline 270K Ohm & $270 \mathrm{~K}$ & 0.1 & Red LED & LED & 0.0958 \\
\hline $1 \mathrm{~K} \mathrm{Ohm}$ & $1 \mathrm{~K}$ & 0.1 & \multicolumn{3}{|l|}{ Ceramic capacitors } \\
\hline 4.7 Ohm & $4 \mathrm{~K} 8$ & 0.1 & $100 \mathrm{P}$ & $100 \mathrm{P}$ & 0.074 \\
\hline $3.9 \mathrm{Ohm}$ & $3 \mathrm{~K} 10$ & 0.1 & $330 \mathrm{P}$ & $330 \mathrm{P}$ & 0.074 \\
\hline $1.5 \mathrm{Ohm}$ & $1 \mathrm{~K} 6$ & 0.1 & $0.1 \mu \mathrm{F}$ & $0.1 \mathrm{UF}$ & 0.074 \\
\hline $2.2 \mathrm{~K} \mathrm{Ohm}(1 \mathrm{~W})$ & $2 \mathrm{~K} 2 / 1 \mathrm{~W}$ & 0.348 & $0.22 \mu \mathrm{F}$ & $0.22 \mathrm{uF}$ & 0.074 \\
\hline Transistors & & & \multicolumn{3}{|c|}{ Electrolytic capacitors } \\
\hline S9014 & 9014 & 0.0157 & $10 \mu \mathrm{F} 50 \mathrm{~V}$ & $10 \mathrm{UF} / 50 \mathrm{~V}$ & 0.259 \\
\hline S9015 & 9015 & 0.0157 & $47 \mu \mathrm{F} 50 \mathrm{~V}$ & $2 \mathrm{X} 47 \mathrm{UF} / 50 \mathrm{~V}$ & 0.553 \\
\hline $\begin{array}{l}2 \text { SD } 882 \text { and } \\
\text { heat sink }\end{array}$ & D882 & 3.02 & $3300 \mu \mathrm{F} 50 \mathrm{~V}$ & $3300 \mathrm{UF} / 50 \mathrm{~V}$ & 10.8 \\
\hline 2SD1047 & D1047 & 4.33 & \multicolumn{3}{|l|}{ Sockets } \\
\hline L7824CV & 7824 & 1.8 & Input (AC24V) & AC24V & 1.81 \\
\hline \multicolumn{3}{|l|}{ Potentiometers } & Output & -OUT+ & 1.49 \\
\hline $\begin{array}{l}\text { Baoter } 3296 \\
(100 \mathrm{~K})\end{array}$ & $100 \mathrm{~K}$ & 0.771 & $24 \mathrm{~V}$ fan connector & FAN 24V & 0.21 \\
\hline $10 \mathrm{~K}$ & $10 \mathrm{~K}$ & 0.415 & & & \\
\hline
\end{tabular}

Numerical and experimental analysis are conducted in three separate phases as shown in Figs. 1a-c; Phase 1 constitutes the bare board (Fig. 1a), Phase 2 is the board with five (5) components attached (Fig. 1b), and Phase 3 the board with fifty-two (52) components attached (Fig. 1c). A nominally identical board having the component penetrations filled with only solder is also investigated as an additional Phase 4 (Fig. 1d).

\section{Experimental Measurements and Numerical Modelling}

\subsection{Experimental Measurements}

Harmonic and random vibration testing were done for each of the four phases shown in Fig. 1. Fig. 2a shows the basic scheme used to generate and measure the response from a harmonic or random excitation on the PCB. An in-house computer program was developed in LabVIEW@ 2018 to generate either a pure sinusoid or band-limited Gaussian white noise. The National Instruments@ module (NIUSB 6211) converted the digital signal to an analog output and a power amplifier sent the signal to an electromagnetic shaker. Both the power amplifier (Model A-120) and shaker (Model DP V009) were manufactured by Data 


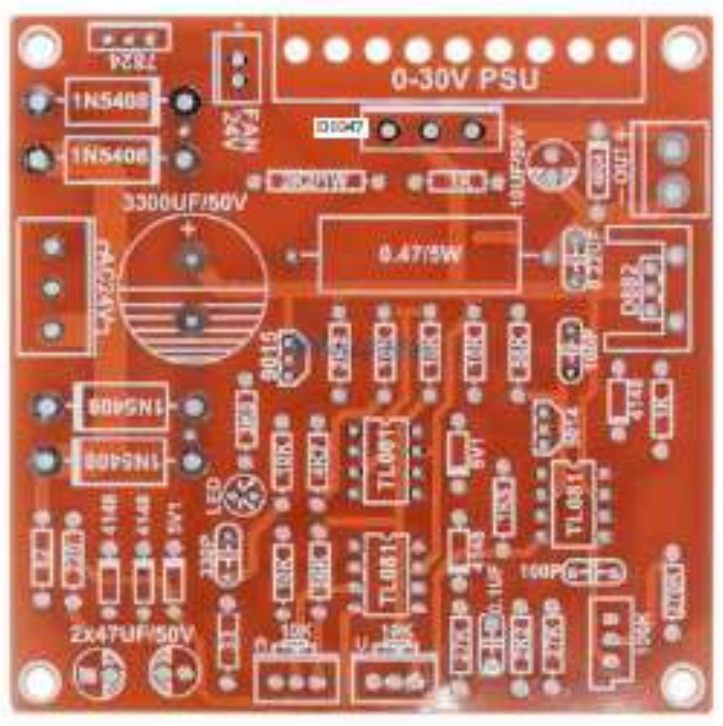

(a)

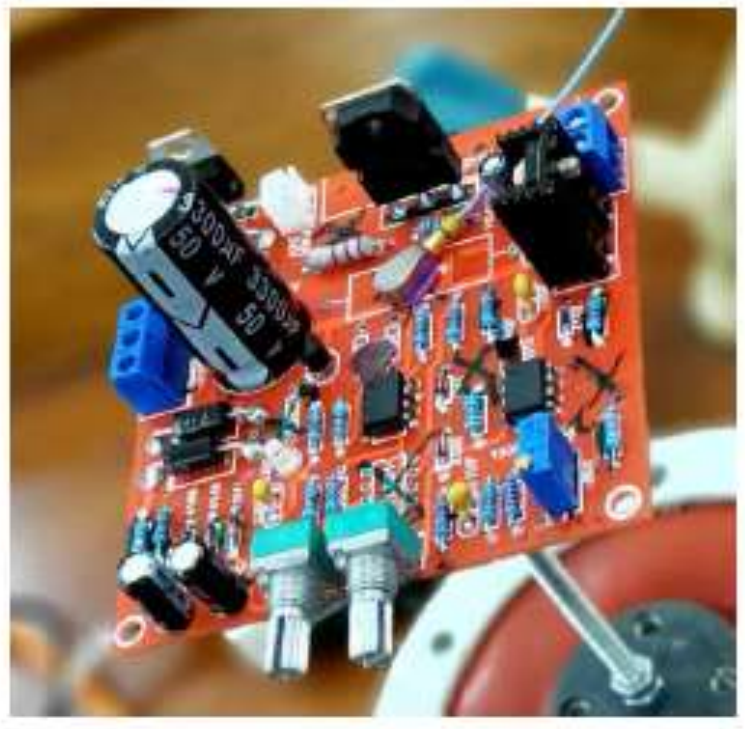

(c)

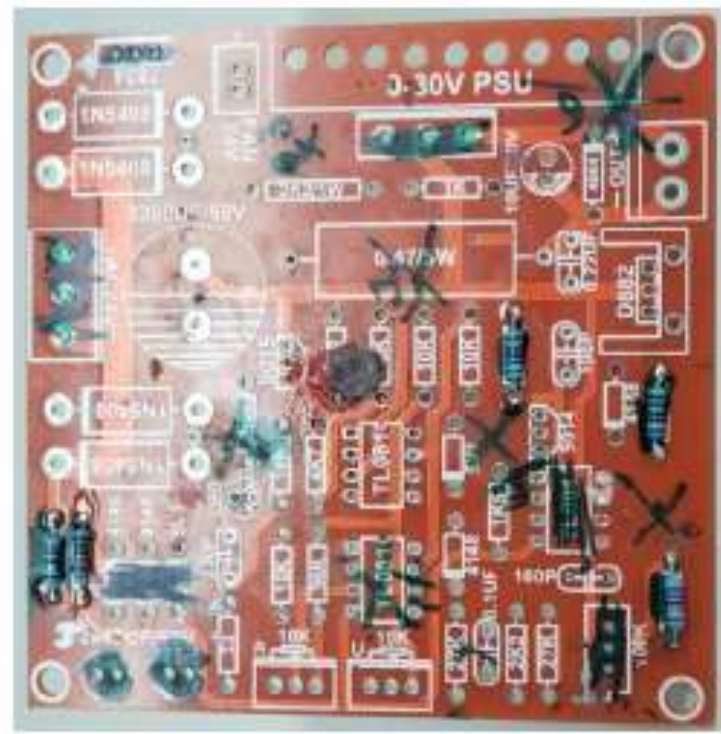

(b)

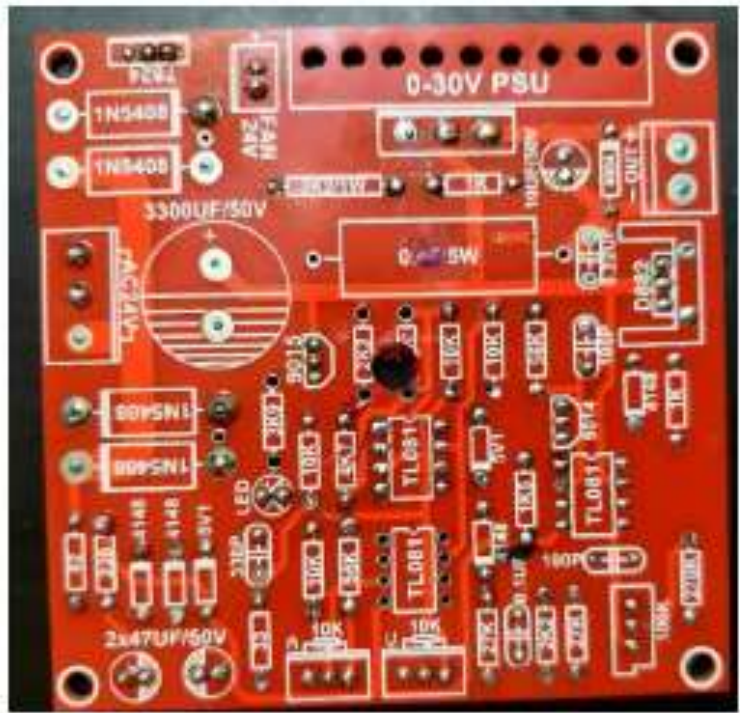

(d)

Figure 1: The phases in which the surface mounted components were attached to the PCB. (a) Phase 1: bare PCB and component tags. (b) Phase 2: five (5) resistors soldered on PCB. (c) Phase 3: fifty-two (52) components soldered on PCB. Accelerometer shown on PCB. (d) Phase 4: PCB having all penetrations designated for components filled with solder.

Physics Corporation. The shaker is rigidly connected to the geometric centre of the PCB via a stinger of diameter, five millimetres $(5 \mathrm{~mm})$. The PCB had a similar sized diametric hole at the centre and was connected to the stinger via an industrial adhesive (Fig. 2b). Vibration measurements of the PCB were taken with an accelerometer manufactured by PCB Piezotronics@ (Model 352A21). The signal conditioner connected to the accelerometer was manufactured by Kistler (Model 5134A). A National Instruments@ module (NIUSB 6009) functioned as an analog to digital converter (ADC) and the accelerometer data was stored and processed using a program written in LabVIEW@ 2018. 
Harmonic testing constituted of driving the shaker with a pure sinusoid and measuring the root mean square (rms) value of the accelerometer response. The ADC sampled data at a rate of $15000 \mathrm{~Hz}$ for a period of two minutes. The frequency of the applied sinusoid was then increased incrementally and the recorded accelerometer data was used to plot a resonance curve or frequency response function of the board. The signal conditioner included an analog antialiasing low pass filter with a cut-off frequency of $1000 \mathrm{~Hz}$. Therefore, all resonance curves were generated between $0-1000 \mathrm{~Hz}$. Harmonic vibration measurements were only taken at Point 1 as shown in Fig. $2 b$.

In the case of random vibration testing, Gaussian white noise in a specific frequency band was used to the drive the shaker. The frequency bands selected always included one resonant mode of the PCB. Generally, the lower and upper frequency band limits depended on the phase of the PCB being considered. For each band of interest rms values were measured using a sampling frequency of $15000 \mathrm{~Hz}$ and a sample time of two (2) minutes. The sample time was shown to be long enough to acquire asymptotic averages.

\subsection{Finite Element Modelling}

Finite element models are generated for the first three phases in Figs. 1a-d using Ansys Mechanical APDL18C. The material and geometric properties used in the FEM are shown in Tables 1 and 2. The bare PCB and solder material which fills the board's penetrations are modelled using Shell181 elements. The copper leads and surface mounted components are modelled using Solid186 elements. The boundary conditions of the PCB are such that the outer edges are all free. The diametric penetration which is rigidly connected to the shaker is treated as being completely fixed (Fig. 3a). The excitation induced by the electrodynamic shaker can then be simulated in the FEM as either a harmonic or random base-displacement. The meshed models for each of the three phases studied is shown in Figs. 3b-d.

The Block Lanczos algorithm in Ansys@ is used to carry out the modal analysis. Harmonic analysis and spectral analysis are also carried out directly in Ansys $\odot$. The damping ratio which is required for harmonic and random analysis in Ansys are experimentally derived from the resonance curves using the half-power bandwidth method [14]. The analytical and numerical response levels are compared for each phase of the PCB studied.

\section{Results and Analysis}

The bare PCB had a single dominant resonant frequency in the range of 0-1000 Hz. The resonance curves obtained both experimentally and numerically are normalized for comparison and are as shown in Fig. 4. The agreement is generally good and the percentage difference between resonant frequency is $1.56 \%$ (Table 3 ). The damping ratio of the mode is calculated as 0.02 . The natural mode shape of the bare PCB can also be visualized experimentally using Chladni's patterns as shown in Fig. 5a. The accumulation of white powder at the nodes bear similar resemblance to that of the mode shape predicted using FEM (Fig. 5b) thereby validating the numerical model. 


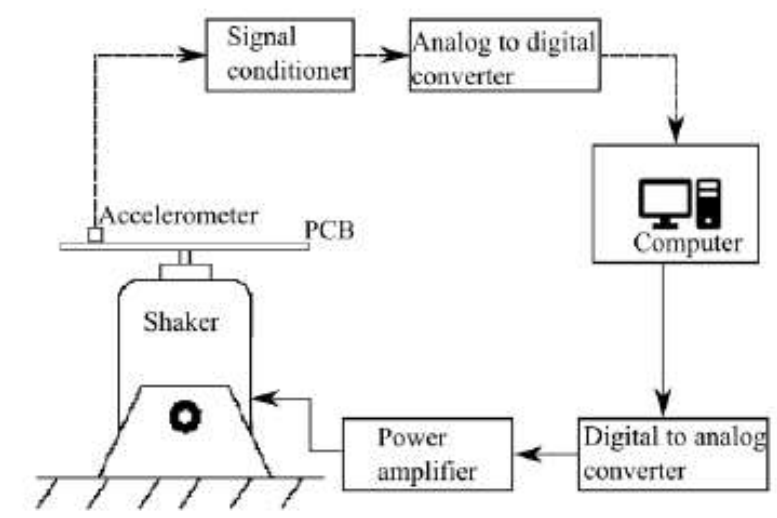

(a)

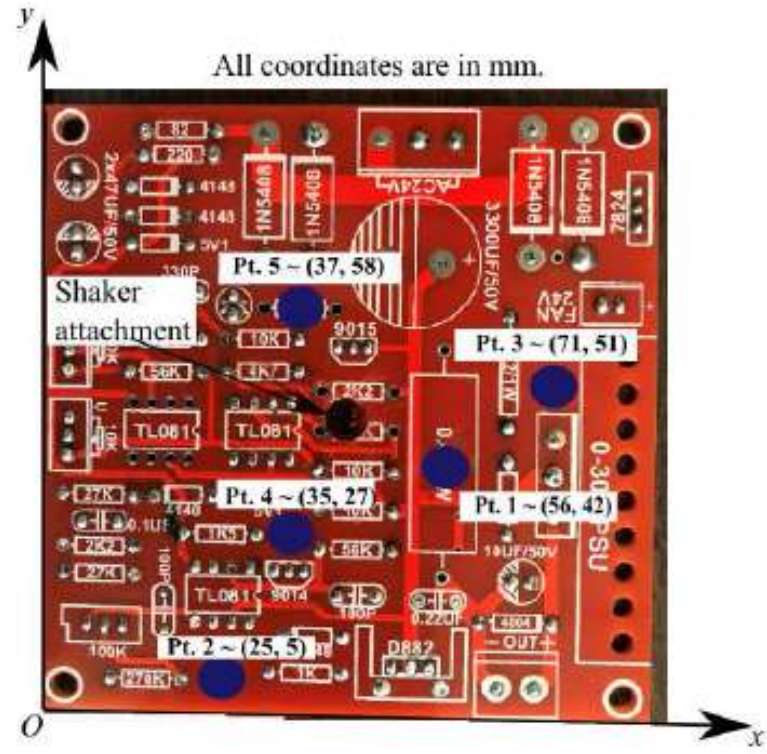

(b)

Figure 2: Vibration excitation, data acquisition and sensor locations on PCB. (a) Vibration excitation and measurement schematic. (b) Measurement locations on PCB with reference to a Cartesian coordinate (x, y) system.

The frequency response of the Phase 2 and 3 configurations are as shown in Figs. 6a and 6b respectively. The agreement between experimental and numerical data are good for both boards. Table 3 indicates that the percentage deviation between experimental and predicted resonant frequencies for both boards are $3.24 \%$ and $1.79 \%$ respectively. Phase 2 shows a decrease of $3.6 \%$ in natural frequency compared to the bare board whilst Phase 3 has a substantially larger increase of $74 \%$. The damping ratio of Phase 2 increases by $50 \%$ to 0.03 and reduces by $65 \%$ to 0.007 for Phase 3 .

The relatively small change in natural frequency from Phase 1 to Phase 2 is reflected when comparing the mode shapes of Fig. $5 \mathrm{~b}$ with that of Fig. $7 \mathrm{a}$ which shows they are generally similar for both phases. A more pronounced change associated with Phase 3 compared to Phase 1 is observed by comparing Fig. 5b with Fig. 7b. In particular, the zone of zero displacement present in Phase 1 and 2 have disappeared in Phase 3. It is also evident that whilst there is a gradual change of displacement in the radially outward direction for Phases 1 and 2; no such pattern exists for Phase 3.

The subsequent analysis indicates that surface mounted components and the associated solder joints can have a significant effect on the dominant natural frequencies, mode shapes and damping on the overall PCB. The components and solder attached to the PCB increase the localized mass and stiffness of the board. Generally, increasing the number of localized masses results in a decrease in the dominant natural frequency [13] whilst an increase in localized stiffness results in a corresponding increase. The mass and stiffness mechanisms for increasing or decreasing the dominant resonant frequency of the board is dependent on the number of soldered components. The effect of the solder material alone is investigated in Phase 4 . In line 


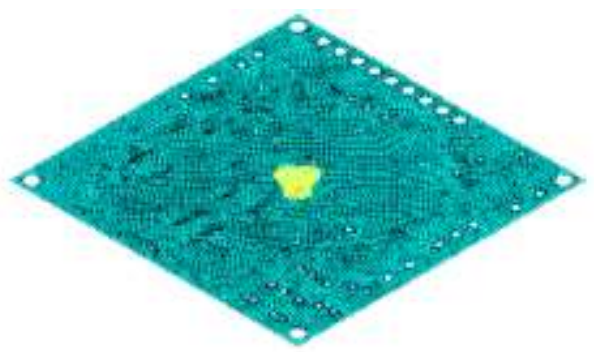

(a)

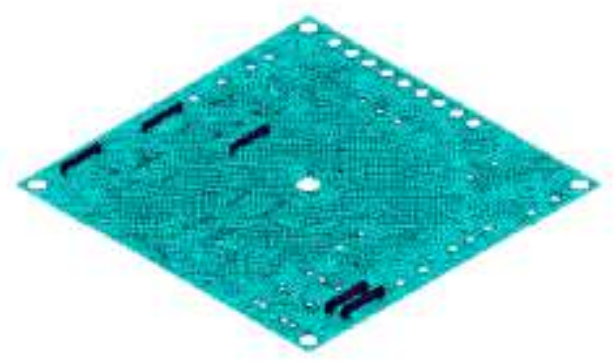

(c)

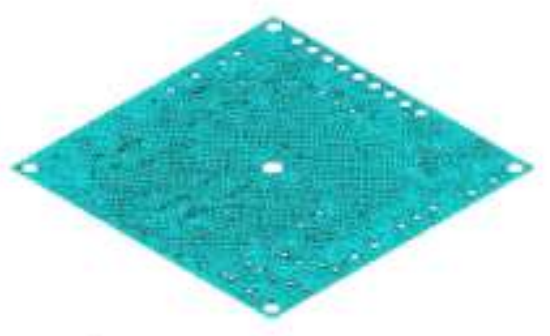

(b)

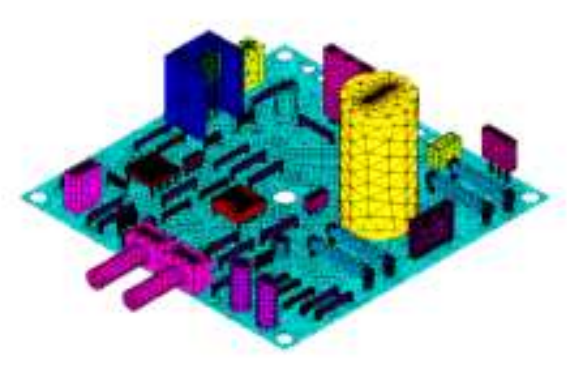

(d)

Figure 3: Finite element models of the PCB and its surface mounted components. The boundary conditions are only indicated in Fig. 3a. (a) Phase 1: Bare circuit board with fixed inner edge. (b) Phase 1: Bare circuit board. (c) Phase 2: five (5) resistors soldered on PCB. (d) Phase 3: fifty-two (52) components soldered on PCB.

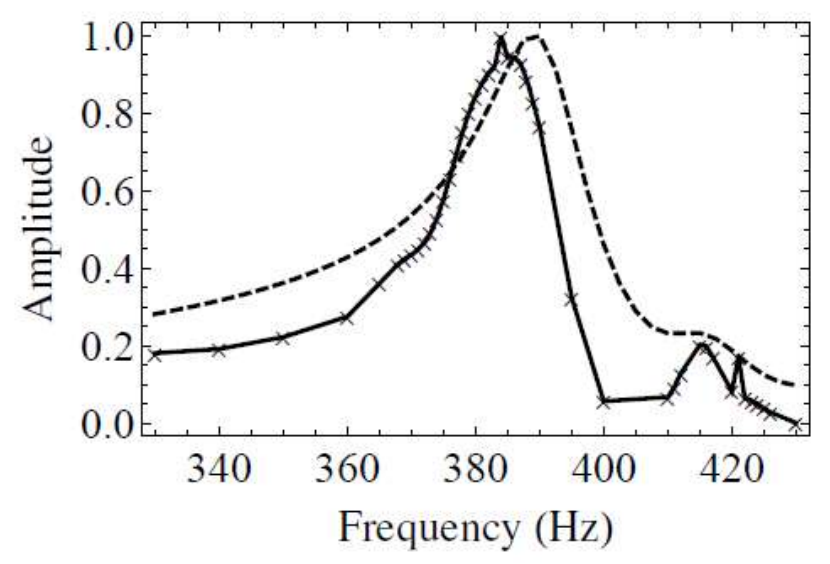

Figure 4: Resonance curves of the PCB in Phase 1. Legend: Experimentally obtained data $(\rightarrow-)$ and numerically (FEM) obtained data (- - -).

Table 3. Resonant frequencies of the PCBs.

\begin{tabular}{llll}
\hline & \multicolumn{3}{c}{ Resonant Frequency $(\mathrm{Hz})$} \\
\hline & Experimental & $\begin{array}{l}\text { Numerical } \\
\text { (FEM) }\end{array}$ & Deviation \\
\hline Phase 1 & 384 & 390 & $1.56 \%$ \\
Phase 2 & 370 & 382 & $3.24 \%$ \\
Phase 3 & 670 & 682 & $1.79 \%$ \\
\hline
\end{tabular}




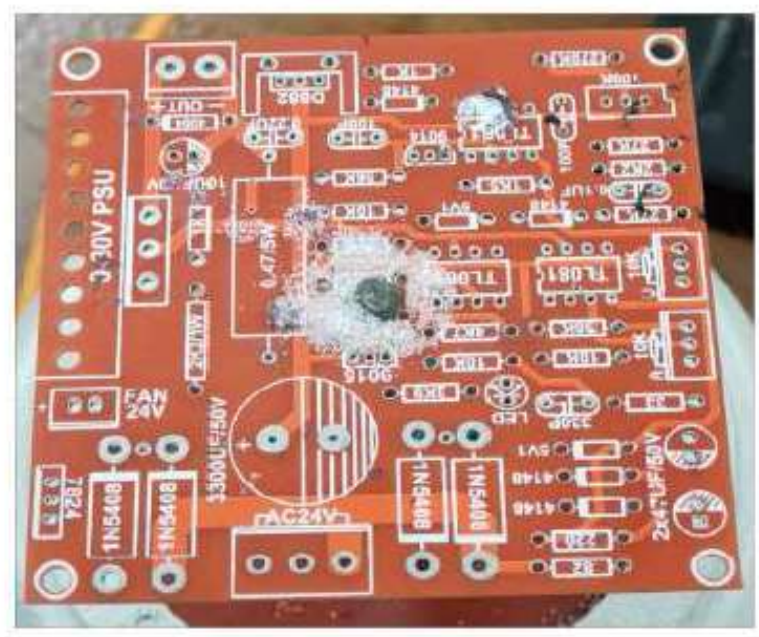

(a)

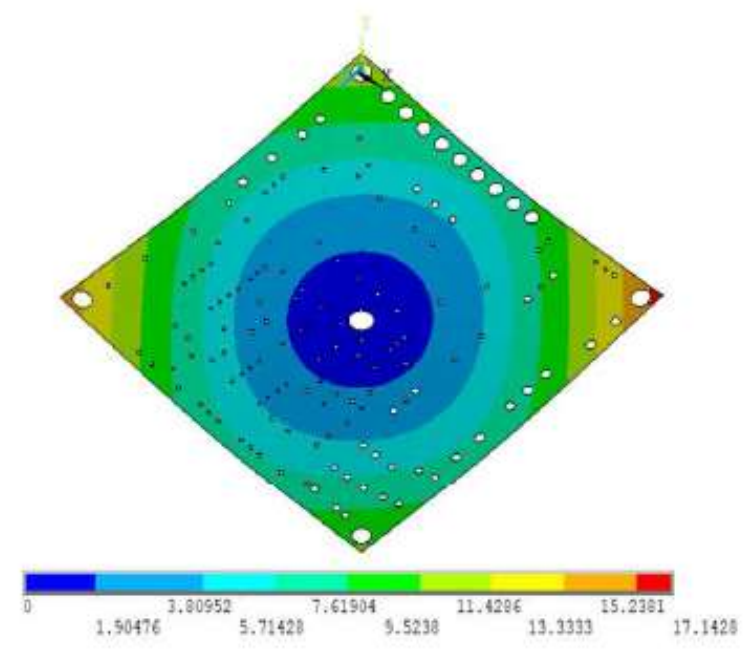

(b)

Figure 5: Mode shape of the Phase 1 PCB. (a) Chladni's pattern for the dominant mode. (b) Numerically determined mode shape.

with our argument, the solder material is itself denser and stiffer than the bare PCB (Table 1). In Fig. 8 the frequency response curves of the bare PCB (Phase 1) and the soldered PCB (Phase 4) are compared. The frequency values in Fig. 8 are normalized by dividing by the resonant frequency of the bare PCB. It is evident that the solder decreases the natural frequency by six percent $(6 \%)$ and the damping loss factor reduces from 0.02 to 0.014 . The increase in mass due to the solder material dominates the increase in local stiffness thereby reducing the value of the natural frequency. This analysis can be extended to Phase 2 to infer that the additional masses of the five resistors also dominated the stiffness in the localized regions, thereby resulting in the decrease of the resonant mode of vibration. In Phase 3 a significantly larger number of surface mounted components increased the mass significantly, however, the stiffness also increased owing to the large number of leads and soldered joints. In this phase the stiffness effects had a much greater effect compared to the mass effect thereby increasing the natural frequency. From a design perspective the foregoing analysis indicates that through the appropriate selection and placement of electronic components on a PCB; the resonant dynamic characteristics may be tailored to a certain extent.

In instances where PCBs are subjected to broadband random excitation, knowing the resonant frequency and mode shapes are important in predicting the average response levels. In line with the methodology presented in Section 3.1, the response at five different points are measured for Phases 1-3 when the board is excited using band-limited Gaussian white noise. The bar-charts in Figs. 9 show the ratio of the measured points relative to point 3 (see Fig. $2 b$ ) for all three phases. The numerically generated results are presented alongside the measured values. There is good agreement between the experimental and numerical results thereby further validating the finite element model. The finite element model can be used to generate the power spectral densities of the induced stresses which can then be incorporated into conventional fatigue life assessment methods to determine the time to failure. 


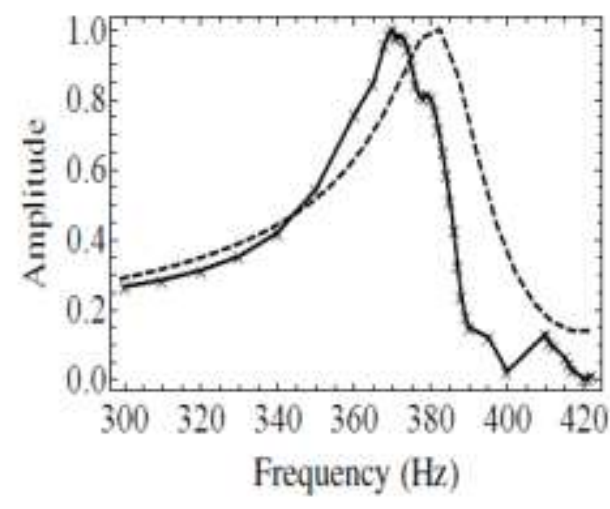

(a)

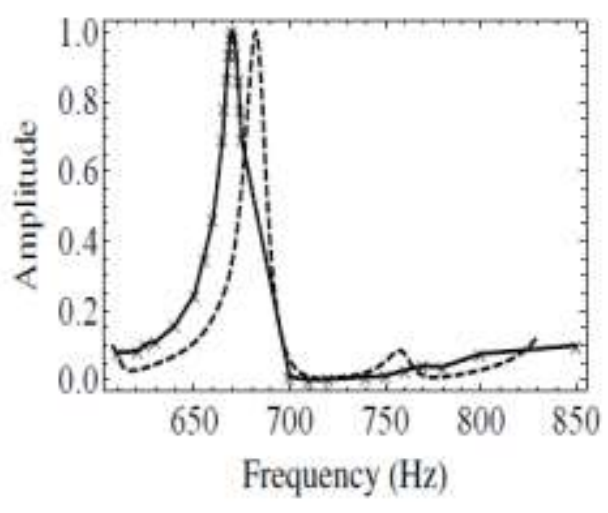

(b)

Figure 4: Resonance curves of the PCB in Phase 1. Legend: Experimentally obtained data $(\rightarrow-)$ and numerically (FEM) obtained data (- - -). (a) Resonance curve of the Phase 2 PCB. (b) Resonance curve of the Phase 3 PCB.

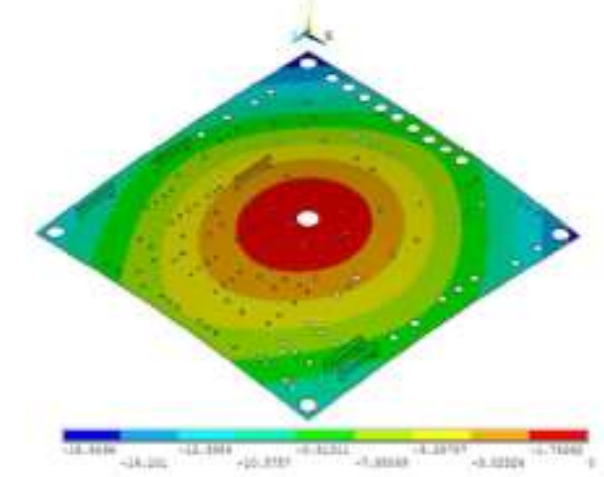

(a)

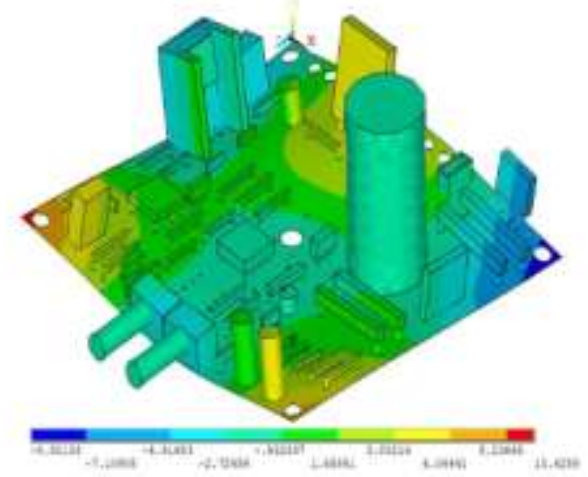

(b)

Figure 7: Mode shapes of the PCB. (a) Dominant mode shape for the Phase 2 PCB. (b) Dominant mode shape for the Phase 3 PCB.

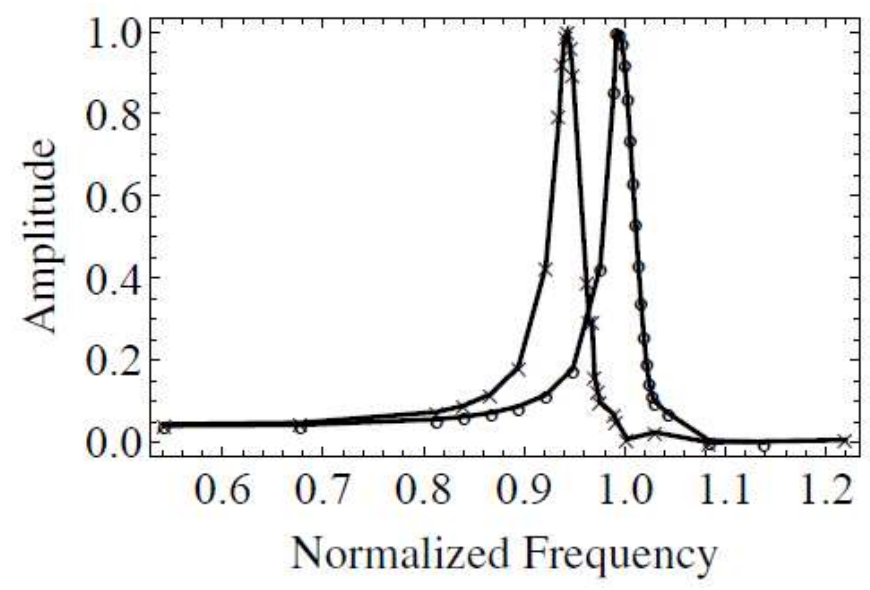

Figure 8: Experimentally obtained resonance curve of the Phase 1 and Phase 4 PCB. Legend: Phase 1 PCB $(\ominus)$ and Phase 4 PCB $(\star \bullet)$. 


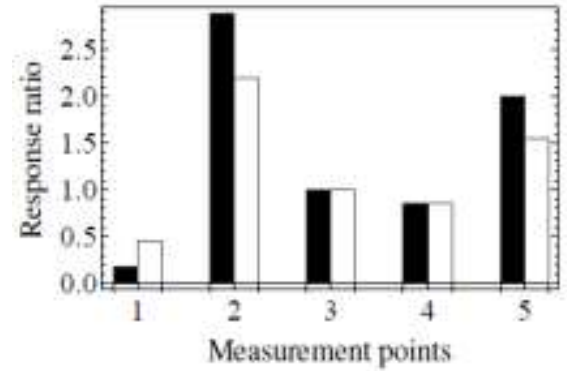

(a)

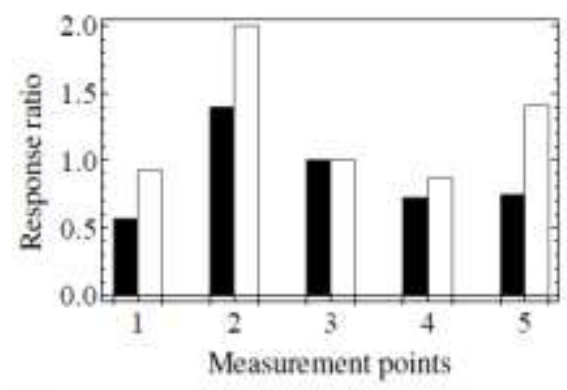

(e)

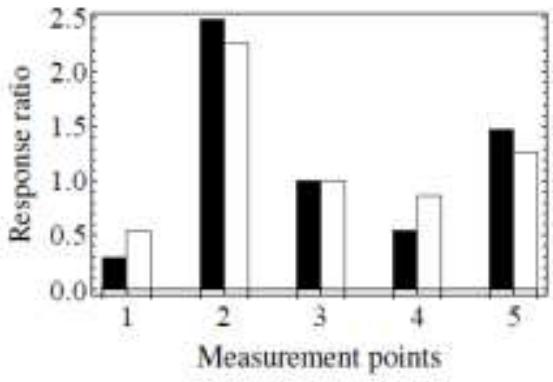

(b)

Figure 9: Random vibration response levels for Phases 1-3. Legend: Experimentally obtained response ratios ( $\boldsymbol{\square}$ ) and numerically obtained response ratios ( $\square$ ). (a) Phase 1 PCB excited with band limited Gaussian white noise between 300-500Hz. (b) Phase 2 PCB excited with band limited

Gaussian white noise between 300-500Hz. (c) Phase 1 PCB excited with band limited Gaussian white noise between 100-1000Hz.

\section{Conclusions}

The geometry, mass and number of electronic components soldered on a PCB will vary from one engineering application to the other. The resonant behaviour of a PCB is influenced by the characteristics and location of the components. In this study the effect of localized component mass and stiffness on the resonant characteristics of a PCB were investigated experimentally and numerically using the FEM. It is shown that for boards with a small number of soldered components the mass effect dominates the stiffness effects and the natural frequency is reduced compared to a bare PCB. The mode shapes of both boards are also shown to be almost identical. For a PCB with a large number of soldered components the increased number of localized stiffness points dominates the localized mass effect and the natural frequency increases significantly. The mode shape for the densely populated board also differs significantly from that of the bare PCB. It is also shown, experimentally, that the effects of the solder material reduce the resonant frequency of a PCB. The study also generates finite element models for three of the four PCB cases studied and good agreement with the experimental results is obtained. The precision of the finite element models and the variation in resonant behaviour associated with varying the number of components on the PCB underscores the potential of designers to gain some control of the dynamic characteristics of the PCB. Intuitively, it is the function which the PCB performs that ultimately defines the components on the board, however, if at the design stage an analyst has flexibility to modify the location or mass of the soldered components, then dynamic tailoring to a certain extent may be achieved. One factor which was excluded from this study but which may also be useful for dynamically tailoring of the PCB is the board's thickness. The work also considered only one type of excitation configuration and hence one set of boundary 
conditions. It is the case, however, that both experimental and numerical analysis can be extended to deal with any arbitrary boundary conditions.

\section{References}

[1] R.S. Li. A methodology for fatigue prediction of electronic components un- der random vibration load, Journal of Electronic Packaging 123 no.4 (2001) 394-400.

[2] E. Suhir. Predicted fundamental vibration frequency of a heavy electronic component mounted on a printed circuit board, Journal of Electronic Packaging 122 no.1 (2000) 3-5.

[3] B. Aytekin, H.N. Ozguven. 2008 Vibration analysis of a simply supported PCB with a component-an analytical approach. In 10th Electronics Packaging Technology Conference (pp. 1178-1183). IEEE

[4] A.V. Burmitskih, A.P. Lebedev, A.A. Levitskiy, M.S. Moskovskih. 2013. Printed Circuit Board vibration analysis using simplified finite element models, 2013 International Siberian Conference on Control and Communications (SIBCON) (pp. 1-4) IEEE.

[5] S. Jayaraman, T. Manish, K. Somashekar and M. Ravindra. Response spectrum analysis of printed circuit boards subjected to shock loads, Procedia Engineering 144 (2016). 1469-1476.

[6] J.E. Luan, T.Y. Tee, E. Pek C.T. Lim. 2003 Modal analysis and dynamic responses of board level drop test, Proceedings of the 5th Electronics Packaging Technology Conference (EPTC 2003) (pp. 233-243).

[7] M.A. Anuar, AA Mat Isa, Z.A.R. Ummi. Modal characteristics study of CEM-1 single-layer printed circuit board using experimental modal analysis, Procedia Engineering 41 (2012): 1360-1366.

[8] Y.S. Chen, C.S. Wang, Y.J. Yang. Combining vibration test with finite element analysis for the fatigue life estimation of PBGA components, Microelectronics reliability 48 (2008): 638-644.

[9] D. Yu, A. Al-Yafawi, S. Park, S. Chung. 2010. Finite element based fatigue life prediction for electronic components under random vibration loading, Proceedings 60th Electronic Components and Technology Conference (ECTC) (pp.188-193).

[10] Y. Zhou, M. Al-Bassyiouni, A. Dasgupta. Harmonic and random vibration durability of SAC305 and Sn37Pb solder alloys, IEEE Transactions on Components and Packaging Technologies 33 (2010): 319328.

[11] J.H. Lau, C.A. Keely. Dynamic characterization of surface-mount component leads for solder joint inspection, IEEE Transactions on Components, Hybrids, and Manufacturing Technology 12 (1989): 594602.

[12] R.A. Amy, G.S. Aglietti, G. Richardson. Accuracy of simplified printed circuit board finite element models. Microelectronics Reliability 50 (2010): 86-97.

[13] T. Wong, K.K. Stevens, G. Wang. Experimental modal analysis and dynamic response prediction of PC boards with surface mount electronic components. Journal of Electronic Packaging 113 (1991): 244249.

[14] W. Thomson. Theory of vibration with applications. Prentice hall; 1993. 